University of Texas Rio Grande Valley

ScholarWorks @ UTRGV

$2-17-2006$

\title{
High-Resolution Studies of Tropolone in the S 0 and S 1 Electronic States: Isotope Driven Dynamics in the Zero-Point Energy Levels
}

John C. Keske

Wei Lin

The University of Texas Rio Grande Valley, wei.lin@utrgv.edu

Wallace C. Pringle

Stewart E. Novick

Thomas A. Blake

See next page for additional authors

Follow this and additional works at: https://scholarworks.utrgv.edu/chem_fac

Part of the Chemistry Commons

\section{Recommended Citation}

Keske, J. C., Lin, W., Pringle, W. C., Novick, S. E., Blake, T. A., \& Plusquellic, D. F. (2006). High-resolution studies of tropolone in the S0 and S1 electronic states: Isotope driven dynamics in the zero-point energy levels. The Journal of Chemical Physics, 124(7), 074309. https://doi.org/10.1063/1.2165652

This Article is brought to you for free and open access by the College of Sciences at ScholarWorks @ UTRGV. It has been accepted for inclusion in Chemistry Faculty Publications and Presentations by an authorized administrator of ScholarWorks@ UTRGV. For more information, please contact justin.white@utrgv.edu,william.flores01@utrgv.edu. 


\section{Authors}

John C. Keske, Wei Lin, Wallace C. Pringle, Stewart E. Novick, Thomas A. Blake, and David F. Plusquellic 


\section{High-resolution studies of tropolone in the $S_{0}$ and $S_{1}$ electronic states: Isotope driven dynamics in the zero-point energy levels}

Cite as: J. Chem. Phys. 124, 074309 (2006); https://doi.org/10.1063/1.2165652

Submitted: 14 November 2005 . Accepted: 16 December 2005 . Published Online: 17 February 2006

John C. Keske, Wei Lin, Wallace C. Pringle, Stewart E. Novick, Thomas A. Blake, and David F. Plusquellic

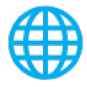

\section{ARTICLES YOU MAY BE INTERESTED IN}

Determination of the proton tunneling splitting of tropolone in the ground state by microwave spectroscopy

The Journal of Chemical Physics 110, 1969 (1999); https://doi.org/10.1063/1.477863

An exploration of electronic structure and nuclear dynamics in tropolone. I. The $\tilde{X}^{1} A_{1}$ ground state

The Journal of Chemical Physics 124, 204307 (2006); https://doi.org/10.1063/1.2200343

Rotation-tunneling analysis of the origin band in the tropolone $\pi^{*} \leftarrow \pi$ absorption system

The Journal of Chemical Physics 120, 4638 (2004); https://doi.org/10.1063/1.1645774

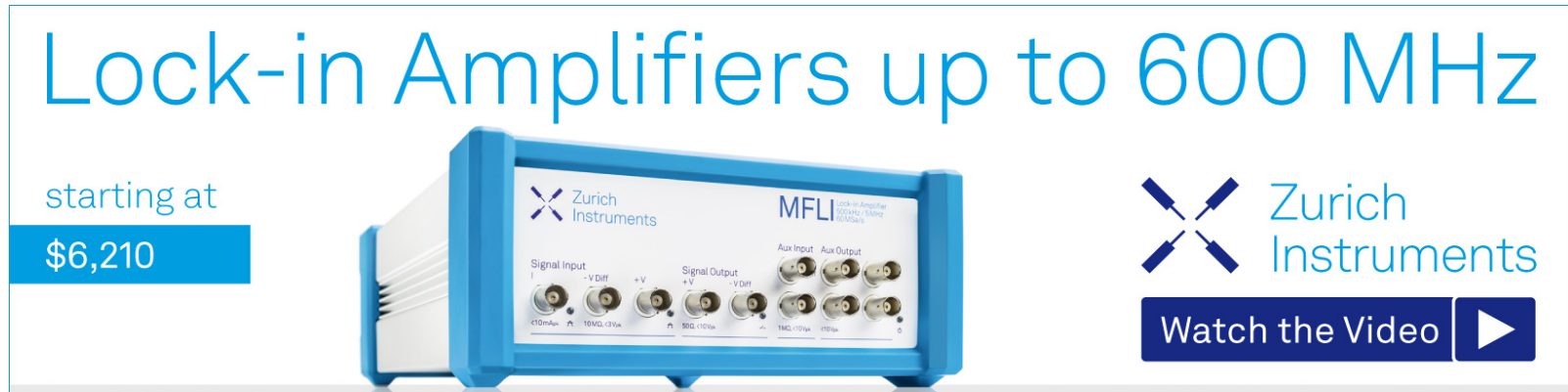




\title{
High-resolution studies of tropolone in the $S_{0}$ and $S_{1}$ electronic states: Isotope driven dynamics in the zero-point energy levels
}

\author{
John C. Keske \\ Optical Technology Division, National Institute of Standards and Technology, Gaithersburg, \\ Maryland 20899-8441
}

Wei Lin, ${ }^{\text {a) }}$ Wallace C. Pringle, and Stewart E. Novick

Wesleyan University, Middletown, Connecticut 06459

Thomas A. Blake

Pacific Northwest National Laboratory, Richland, Washington 99352

David F. Plusquellic ${ }^{\text {b) }}$

Optical Technology Division, National Institute of Standards and Technology, Gaithersburg, Maryland 20899-8441

(Received 14 November 2005; accepted 16 December 2005; published online 17 February 2006)

Rotationally resolved microwave (MW) and ultraviolet (UV) spectra of jet-cooled tropolone have been obtained in $S_{0}$ and $S_{1}$ electronic states using Fourier-transform microwave and UV-laser/ molecular-beam spectrometers. In the ground electronic state, the MW spectra of all heavy-atom isotopomers including one ${ }^{18} \mathrm{O}$ and four ${ }^{13} \mathrm{C}$ isotopomers were observed in natural abundance. The OD isotopomer was obtained from isotopically enriched samples. The two lowest tunneling states of each isotopomer except ${ }^{18} \mathrm{O}$ have been assigned. The observed inversion splitting for the OD isotopomer is $1523.227(5) \mathrm{MHz}$. For the asymmetric ${ }^{13} \mathrm{C}$ structures, the magnitudes of tunneling-rotation interactions are found to diminish with decreasing distance between the heavy atom and the tunneling proton. In the limit of closest approach, the $0^{+}$state of ${ }^{18} \mathrm{O}$ was well fitted to an asymmetric rotor Hamiltonian, reflecting significant changes in the tautomerization dynamics. Comparisons of the substituted atom coordinates with theoretical predictions at the MP2/ aug-cc-pVTZ level of theory suggest the localized $0^{+}$and $0^{-}$wave functions of the heavier isotopes favor the $\mathrm{C}-\mathrm{OH}$ and $\mathrm{C}=\mathrm{O}$ forms of tropolone, respectively. The only exception occurs for the ${ }^{13} \mathrm{C}-\mathrm{OH}$ and ${ }^{13} \mathrm{C}=\mathrm{O}$ structures which correlate to the $0^{-}$and $0^{+}$states, respectively. These preferences reflect kinetic isotope effects as quantitatively verified by the calculated zero-point energy differences between members of the asymmetric atom pairs. From rotationally resolved data of the $0^{+} \leftarrow 0^{+}$and $0^{-} \leftarrow 0^{-}$bands in $S_{1}$, line-shape fits have yielded Lorentzian linewidths that differ by $12.2(16) \mathrm{MHz}$ over the $19.88(4) \mathrm{cm}^{-1}$ interval in $S_{1}$. The fluorescence decay rates together with previously reported quantum yield data give nonradiative decay rates of 7.7(5) $\times 10^{8}$ and $8.5(5)$ $\times 10^{8} \mathrm{~s}^{-1}$ for the $0^{+}$and $0^{-}$levels of the $S_{1}$ state of tropolone. (C) 2006 American Institute of Physics. [DOI: 10.1063/1.2165652]

\section{INTRODUCTION}

Tropolone (2-hydroxy-2,4,6-cycloheptatriene-1-one) has served as a prototypical molecule for proton transfer. Facile intramolecular proton transfer occurs between the two carbonyl oxygens during the tautomerization of the seven-member carbon ring. A schematic diagram of the potential-energy surfaces associated with the proton transfer coordinate is shown in Fig. 1. The symmetric double-minimum potentials have sufficiently low barriers that tunneling splittings are resolved in both the $S_{0}\left(\widetilde{x}^{1} A_{1}\right)$ and $S_{1}\left(\widetilde{A}^{1} B_{2}\right)$ electronic states at modest resolution. The vibrational wave functions of the two

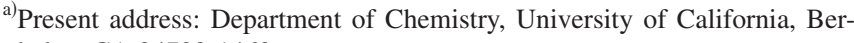
keley, CA 94720-1460.

${ }^{b)}$ Electronic mail: david.plusquellic@nist.gov
}

lowest-energy tunneling components, designated as the $0^{+}$ and $0^{-}$states, have $A_{1}$ and $B_{2}$ symmetry in the $C_{2 v}$ pointgroup symmetry of the transition state.

Tropolone has been the subject of numerous experimental ${ }^{1-7}$ and theoretical ${ }^{8-12}$ studies in matrix and gasphase environments. Tanaka et al. have accurately determined tunneling splitting to be $0.97379995(87) \mathrm{cm}^{-1}$ from jet-cooled microwave (MW) studies of the ground state. ${ }^{2}$ Doublets arising from the difference in the tunneling splittings in $S_{1}$ and $S_{0}$ were first reported by Alves and Hollas in the vapor-phase absorption spectrum. ${ }^{13,14}$ More recently, high-resolution degenerate four-wave mixing experiments of bulk-gas-phase samples ${ }^{15}$ have been made near the $S_{1}-S_{0}\left(\pi^{*} \leftarrow \pi\right)$ electronic origin. Accurate excited-state parameters analogous to those obtained for the ground state have been reported. From these results, the tunneling split- 


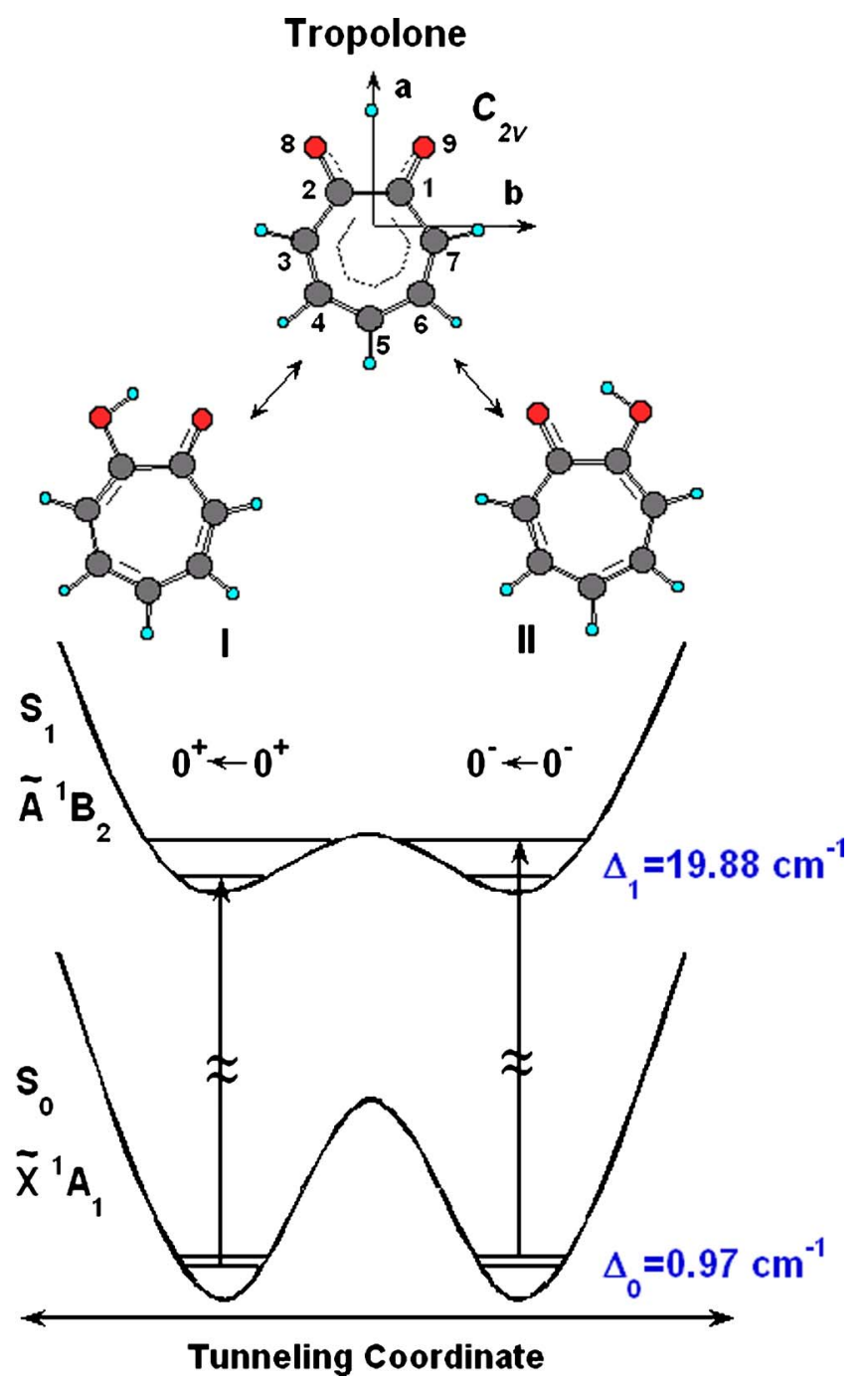

FIG. 1. Schematic illustration of the $S_{0}$ and $S_{1}$ surfaces of tropolone along the proton transfer coordinate.

ting in $S_{1}\left(\Delta_{1}\right)$ undergoes a drastic increase to $>19 \mathrm{~cm}^{-1}$, illustrating the importance of the $\pi$ electron system on the tautomerization dynamics.

Additional studies have examined the dependence of the tunneling splitting on the vibrational mode excited in both $S_{0}$ (Refs. 4, 7, 11, and 16) and $S_{1} \cdot{ }^{17-19}$ These investigations have shown that the splitting decreases with increasing vibrational excitation along other vibrational coordinates, highlighting the sensitivity of the tunneling dynamics to nuclear displacements of the frame. For example, it was found that vibrations involving the motion of the oxygen atoms and out-of-plane modes affecting the planarity of the molecule have the largest impact on the proton-tunneling dynamics. The influence of the tropolone-ring substitutions on the tunneling splittings has also been investigated using laser-induced fluorescence (LIF) techniques for several symmetric and asymmetric analogs. ${ }^{9,20-23}$ Like tropolone, tunneling doublets are observed in the spectra of the symmetric forms. The protontunneling dynamics is, however, effectively quenched with asymmetric substitution as a result of the loss of symmetry along the tunneling coordinate.

In this study, we have obtained rotationally resolved spectra of tropolone in the ground and first electronically excited states. In $S_{0}$, the analysis of the symmetric isotopomers $\left({ }^{12} \mathrm{C},{ }^{13} \mathrm{C}_{5}\right.$, and OD forms) has permitted an estimate of the ground-state tunneling barrier. For the other asymmetric heavy-atom isotopomers, we find that the tautomerization dynamics becomes rapidly quenched with increasing proximity of the isotopic label to the tunneling proton, causing, in effect, a preferential selectivity for one of the two resonance structures in each tunneling state. The selectivity is shown to depend on the substituted atom position and reflect kinetic isotope effects that are quantitatively explained in terms of the calculated zero-point energies. In the $S_{1}$ state, the fluorescence lifetimes have been used to estimate the radiative and nonradiative decay rates of tropolone and their dependence on tunneling state.

\section{EXPERIMENT}

Pure rotational spectra of tropolone and its isotopomers were obtained using two different Fourier-transform microwave spectrometers. ${ }^{24}$ In the NIST spectrometer, tropolone ( $>95 \%$ purity) was resistively heated to $110{ }^{\circ} \mathrm{C}$ in a reservoir nozzle and expanded with a $\mathrm{He} / \mathrm{Ne}$ gas mixture through a $1.2 \mathrm{~mm}$ pulsed nozzle operating at $6 \mathrm{~Hz}$. For each scan, 30 free-induction decays were averaged and Fourier transformed at stepped intervals of $0.5 \mathrm{MHz}$. In the Wesleyan spectrometer, the tropolone or deuterated tropolone sample was placed in a tube directly upstream of the $0.5 \mathrm{~mm}$ pulsed nozzle and $1 \mathrm{~atm}$ of a $\mathrm{He} / \mathrm{Ne}$ gas mixture flowed over the unheated sample and into the nozzle. For each scan, 50 shots at $10 \mathrm{~Hz}$ were averaged and transformed at intervals of 0.3 $\mathrm{MHz}$.

Fluorescence excitation spectra of the $S_{1} \leftarrow S_{0}$ transitions of tropolone near $370 \mathrm{~nm}$ were measured at NIST using a UV-laser/molecular-beam spectrometer described in detail elsewhere. ${ }^{25}$ Briefly, an $\mathrm{Ar}^{+}$-pumped cw ring Ti-sapphire laser generated $\approx 500 \mathrm{~mW}$ of narrow-band light $(\approx 500 \mathrm{kHz})$ near $740 \mathrm{~nm}$. Approximately $5 \mathrm{~mW}$ of the UV light at 370 $\mathrm{nm}$ were generated in an external resonant cavity containing a lithium borate crystal. Tropolone was heated to $120^{\circ} \mathrm{C}$ in a three-chamber quartz source. The vapor was mixed with 29 $\mathrm{kPa}(220 \mathrm{Torr})$ of $\mathrm{Ar}$ and expanded into a source chamber through a $125 \mu \mathrm{m}$ nozzle. The molecular beam was skimmed and crossed at right angles with a slightly focused UV beam $18 \mathrm{~cm}$ downstream of the source. Laser-induced fluorescence at the beam crossing was collected with $20 \%$ efficiency using two spherical mirrors and detected using a photomultiplier and computer-interfaced photon counter. The Doppler-limited resolution of the spectrometer at $330 \mathrm{~nm}$ was $18 \mathrm{MHz}{ }^{26}$ Relative frequency calibration was performed using a HeNe-stabilized reference cavity, ${ }^{25}$ and absolute frequencies were obtained using a wavemeter accurate to $\pm 0.02 \mathrm{~cm}^{-1}$.

\section{RESULTS}

\section{A. The $0^{+}$and $0^{-}$ground states of the ${ }^{13} \mathrm{C}$ isotopomers of tropolone}

The schematic diagram of the proton-tunneling surfaces of tropolone along the symmetric double-minimum poten- 
TABLE I. $S_{0}$ rotational constants of the parent isotopomer of tropolone obtained from fits of the FTMW spectra.

\begin{tabular}{|c|c|c|}
\hline & \multicolumn{2}{|c|}{${ }^{12} \mathrm{C}$} \\
\hline & $0^{+}$ & $0^{-}$ \\
\hline$A(\mathrm{MHz})$ & $2743.08527(17)^{\mathrm{a}}$ & $2742.71137(18)$ \\
\hline$B(\mathrm{MHz})$ & $1659.891305(85)$ & $1659.864555(98)$ \\
\hline$C(\mathrm{MHz})$ & $1034.383541(65)$ & $1034.324106(61)$ \\
\hline$\Delta I\left(u \AA^{2}\right)$ & -0.122661 & -0.124608 \\
\hline$\Delta_{J}(\mathrm{kHz})$ & $0.04636(75)$ & $0.04606(90)$ \\
\hline$\Delta_{J k}(\mathrm{kHz})$ & $0.0968(22)$ & $0.1014(29)$ \\
\hline$\Delta_{K}(\mathrm{kHz})$ & $0.1430(82)$ & $0.1699(99)$ \\
\hline$\delta_{J}(\mathrm{kHz})$ & $0.01605(31)$ & $0.01594(42)$ \\
\hline$\delta_{K}(\mathrm{kHz})$ & $0.1104(17)$ & $0.1078(24)$ \\
\hline$F_{a b}(\mathrm{MHz})^{\mathrm{b}}$ & $\pm 16.45687(11)$ & $\pm 16.45687(11)$ \\
\hline$\Delta_{0}(\mathrm{MHz})^{\mathrm{b}}$ & $29193.7969(11)$ & $29193.7969(11)$ \\
\hline$a$-type assigned ${ }^{\mathrm{c}}$ & 67 & 59 \\
\hline $0^{-} \leftarrow 0^{+}$assigned $^{c}$ & 27 & 27 \\
\hline$\sigma(\mathrm{kHz})$ & 1.5 & 1.5 \\
\hline$\sigma(\mathrm{kHz})^{\mathrm{d}}$ & 13600 & 13600 \\
\hline
\end{tabular}

${ }^{\mathrm{a}}$ Uncertainties are type $\mathrm{A}, k=1$, and correspond to $1 \sigma$ standard deviations (Ref. 29).

${ }^{\mathrm{b}} F_{a b}$ and $\Delta_{0}$ change by 0.00013 and 0.034 when all level-crossing transitions are excluded.

${ }^{c}$ Experimental uncertainty of all assigned lines is $2 \mathrm{kHz}$.

${ }^{\mathrm{d}}$ Observed minus calculated standard deviation of fits that exclude $F_{a b}$ and $\Delta_{0}$ and the $0^{-} \leftarrow 0^{+}$transitions.

tials in $S_{0}$ and $S_{1}$ is shown in Fig. 1. The microwave spectrum of the $0^{+}$and $0^{-}$states of ${ }^{12} \mathrm{C}$ tropolone has been previously reported by Tanaka et al. ${ }^{2}$ Significant perturbations of the pure rotational transition energies were observed for a wide range of rotational levels from tunneling-rotation interactions between the $0^{+}$and $0^{-}$states, resulting in line position shifts of up to $20 \mathrm{MHz}$ for some of the highest $J$ levels examined. The $0^{+}$and $0^{-}$rotational constants, tunneling splitting $\Delta_{0}$, and the tunneling-rotation interaction term $F_{a b}\left(P_{b} P_{a}+P_{a} P_{b}\right)$ have been well determined from their data. [Here, the small correction factor $F_{J}$ in $\left\{F+F_{J} P^{2}\right\}\left(P_{b} P_{a}\right.$ $\left.+P_{a} P_{b}\right)$ has been ignored. $\left.{ }^{27}\right]$

We have remeasured the Fourier-transform microwave (FTMW) spectrum of tropolone over the range from 11 to 20 $\mathrm{GHz}$. For the parent $\left({ }^{12} \mathrm{C}\right)$ isotopomer, 118 new assignments have been added to the data set of Tanaka et $a l^{2}$ The assigned line set consists of 67 and 59 pure rotational $a$-type transitions of the $0^{+}$and $0^{-}$states, respectively, and $27 b$-type torsion-rotation transitions connecting the $0^{+}$to the $0^{-}$state. A least-squares fit of these transitions in Pickett's reduced axis system ${ }^{27}$ using SPFIT (Ref. 28) has allowed us to refine the rotational constants as well as to include all five of the quartic centrifugal distortion constants. These results are given in Table I. In this table and elsewhere, the parameter uncertainties $^{29}$ are type $\mathrm{A}, k=1$, and correspond to $1 \sigma$ standard deviations unless otherwise noted.

The signal-to-noise ratio of tropolone was sufficiently high that transitions of the ${ }^{13} \mathrm{C}$ and ${ }^{18} \mathrm{O}$ isotopomers were observed in their natural abundances of $1.1 \%$ and $0.2 \%$, respectively. The observed spectrum is shown at the top of Fig. 2 where transitions from the parent have been removed. In tropolone, there are nine heavy atoms to isotopically substi-

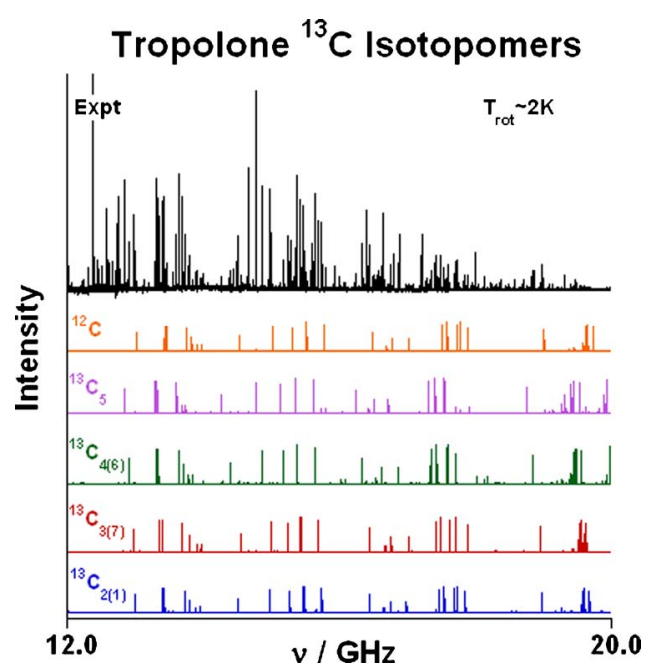

FIG. 2. FTMW spectrum of tropolone (top) with transitions from the parent isotopomer removed. Simulated spectra of the parent and ${ }^{13} \mathrm{C}$ isotopomers are shown in the lower traces.

tute and therefore nine additional states are expected. This number would be expected to double if, like the parent, both tunneling states are observed. However, from Fig. 1, the structural features that distinguish, for example, ${ }^{13} \mathrm{C}_{1}$ and ${ }^{13} \mathrm{C}_{2}$ in structure I are interconverted in structure II. Therefore, the ${ }^{13} \mathrm{C}_{1}$ and ${ }^{13} \mathrm{C}_{2}$ isotopomers will correlate to the two lowest-energy tunneling states, $0^{+}$and $0^{-}$, or vise versa, where the state labels are retained for clarity (and the implied parity of the symmetric forms is no longer valid). The same holds true for the other three asymmetric pairs: ${ }^{13} \mathrm{C}_{3(7)},{ }^{13} \mathrm{C}_{4(6)}$, and ${ }^{18} \mathrm{O}_{8(9)}$. From these considerations, ten states are expected, eight for the asymmetric atoms, and two more for the symmetry position, ${ }^{13} \mathrm{C}_{5}$. From our data, only nine states were observed including the four pairs of ${ }^{13} \mathrm{C}$ states plus the $0^{+}$level of ${ }^{18} \mathrm{O}$ obtained under colder expansion conditions. Both tunneling states of the OD isotopomer were also obtained from enriched samples. The 11 parameter sets obtained from the fits using SPFIT are given in Tables II-IV. Simulated spectra of the four ${ }^{13} \mathrm{C}$ isotopomers are shown below the experimental trace in Fig. 2.

Correlations between the specific locations of the heavyatom isotopes in the structure of tropolone and the parameter sets are easily drawn from simple comparisons of the rotational constants with those of the parent isotopomer, ignoring for the moment small changes in the principal-axis orientation with ${ }^{13} \mathrm{C}$ or ${ }^{18} \mathrm{O}$ substitution. No attempt is made at this time to distinguish between the two subscript labels. For example, the $A$ constants of the ${ }^{12} \mathrm{C}$ data in Table I are nearly identical with those of ${ }^{13} \mathrm{C}$ data in Table II indicating that the isotope is on or near the $a$ axis as is the case only for the $\mathrm{C}_{5}$ position. Likewise, the ${ }^{13} \mathrm{C}_{3(7)}$ position is near the $b$ axis, identifying it with the second data set in Table III. The three remaining identifications are made based on the differences in the $B$ rotational constants as follows. Although of similar distance from the $b$ axis, the $\mathrm{O}_{8(9)}$ atom is heavier than $\mathrm{C}_{4(6)}$, associating the former with the second data set in Table IV. The remaining correlations are easily made from similar arguments that distinguish between the $\mathrm{C}_{1(2)}$ and $\mathrm{C}_{4(6)}$ posi- 
TABLE II. $S_{0}$ rotational constants of the symmetric OD and ${ }^{13} \mathrm{C}_{5}$ isotopomers of tropolone obtained from fits of the FTMW spectra.

\begin{tabular}{|c|c|c|c|c|}
\hline & \multicolumn{2}{|c|}{ OD } & \multicolumn{2}{|c|}{${ }^{13} \mathrm{C}_{5}$} \\
\hline & $0^{+}$ & $0^{-}$ & $0^{+}$ & $0^{-}$ \\
\hline$A(\mathrm{MHz})$ & $2739.153(3)^{\mathrm{a}}$ & 2739.143(3) & $2743.1319(30)$ & $2742.7587(36)$ \\
\hline$B(\mathrm{MHz})$ & $1624.4302(5)$ & $1624.4283(5)$ & $1631.53149(22)$ & $1631.50447(34)$ \\
\hline$C(\mathrm{MHz})$ & $1019.8953(3)$ & $1019.8903(3)$ & $1023.30807(32)$ & $1023.24987(37)$ \\
\hline$\Delta I\left(u \AA^{2}\right)$ & -0.093005 & -0.091613 & -0.12384 & -0.12595 \\
\hline$\Delta_{J}(\mathrm{kHz})$ & $0.0092(30)$ & $0.0093(26)$ & $0.0420(27)$ & $0.0417(30)$ \\
\hline$\Delta_{J K}(\mathrm{kHz})$ & $0.23(8)$ & $0.16(6)$ & 0.0968 & 0.1014 \\
\hline$\Delta_{K}(\mathrm{kHz})$ & $1.7(6)$ & $2.5(5)$ & 0.1430 & 0.1699 \\
\hline$\delta_{J}(\mathrm{kHz})$ & $\ldots$ & $\ldots$ & 0.01605 & 0.01594 \\
\hline$\delta_{K}(\mathrm{kHz})$ & $\ldots$ & $\ldots$ & $0.154(27)$ & $0.135(26)$ \\
\hline$F_{a b}(\mathrm{MHz})$ & $\pm 24.089(2)$ & $\pm 24.089(2)$ & $\pm 15.88909(92)$ & $\pm 15.88909(92)$ \\
\hline$\Delta_{0}(\mathrm{MHz})$ & $1523.227(5)$ & $1523.227(5)$ & $29079.0765(69)$ & $29079.0765(69)$ \\
\hline$a$-type assigned $^{\mathrm{b}}$ & 26 & 27 & 24 & 19 \\
\hline $0^{-} \leftarrow 0^{+}$assigned $^{\mathrm{b}}$ & 5 & 5 & 4 & 4 \\
\hline$\sigma(\mathrm{kHz})$ & 5.4 & 5.4 & 1.5 & 1.5 \\
\hline$\sigma(\mathrm{kHz})^{\mathrm{c}}$ & 3746 & 3746 & 5190 & 5190 \\
\hline
\end{tabular}

${ }^{\mathrm{a}}$ Uncertainties are type A, $k=1$, or $1 \sigma$ (Ref. 29).

${ }^{\mathrm{b}}$ Experimental uncertainties of all assigned lines are $2 \mathrm{kHz}$ for the OD and $4 \mathrm{kHz}$ for the ${ }^{13} \mathrm{C}_{5}$ isotopomers.

${ }^{c}$ Observed minus calculated standard deviation of fits that exclude $F_{a b}, \Delta_{0}$ and the $0^{-} \leftarrow 0^{+}$transitions.

tions. The overall intensities of the ${ }^{13} \mathrm{C}_{1(2)},{ }^{13} \mathrm{C}_{3(7)}$, and ${ }^{13} \mathrm{C}_{4(6)}$ spectra were roughly twofold greater than those of the

${ }^{13} \mathrm{C}_{5}$ isotopomer as expected from the ratio of structures having two positions for asymmetric substitution compared to the single symmetric form.

For the nine heavy-atom isotopomers, the standard deviations of the fits are better than $3 \mathrm{kHz}$. The standard deviation of the OD fit increases somewhat to $5 \mathrm{kHz}$ as a result of unassigned and overlapping deuterium nuclear quadrupole hyperfine splittings. Except for the ${ }^{18} \mathrm{O}_{8(9)}$ spectrum which was well fitted to a standard asymmetric rotor Hamiltonian, the tunneling-rotation interaction terms $F_{a b}$ and splittings $\Delta_{0}$ have been well determined. For the ${ }^{13} \mathrm{C}_{5}$ and ${ }^{13} \mathrm{C}_{4(6)}$ isotopomers, the locations of several $0^{-} \leftarrow 0^{+} b$-type or levelcrossing transitions were predicted and assigned following initial fits of the pure rotational transitions of the $0^{+}$and $0^{-}$ states. The assignment of these nominally forbidden transitions substantially improved the fit quality of the tunneling parameters. However, even in their absence, accurate determinations of the tunneling parameters for the ${ }^{13} \mathrm{C}_{3(7)}$ and ${ }^{13} \mathrm{C}_{2(1)}$ isotopomers have been possible as evident from the following example.

The dependence of the tunneling parameters on the observed rotational energy levels is readily apparent for a two-

TABLE III. $S_{0}$ rotational constants of the asymmetric ${ }^{13} \mathrm{C}$ isotopomers of tropolone obtained from fits of the FTMW spectra.

\begin{tabular}{|c|c|c|c|c|}
\hline & \multicolumn{2}{|c|}{${ }^{13} \mathrm{C}_{4(6)}$} & \multicolumn{2}{|c|}{${ }^{13} \mathrm{C}_{3(7)}$} \\
\hline & $0^{+}$ & $0^{-}$ & $0^{+}$ & $0^{-}$ \\
\hline$A(\mathrm{MHz})$ & $2720.4853(17)^{\mathrm{a}}$ & $2720.3087(30)$ & $2706.8435(47)$ & $2706.1020(88)$ \\
\hline$B(\mathrm{MHz})$ & $1644.39289(24)$ & $1644.31108(38)$ & $1659.33137(89)$ & $1659.1377(15)$ \\
\hline$C(\mathrm{MHz})$ & $1025.15220(25)$ & $1025.09981(44)$ & $1028.98154(48)$ & $1028.8126(15)$ \\
\hline$\Delta I\left(\mathrm{u} \AA^{2}\right)$ & -0.12318 & -0.12533 & -0.12717 & -0.13326 \\
\hline$\Delta_{J}(\mathrm{kHz})$ & $0.0416(22)$ & $0.0462(64)$ & $0.0658(88)$ & $0.108(15)$ \\
\hline$\Delta_{J K}(\mathrm{kHz})$ & $0.100(19)$ & $0.068(23)$ & $0.510(44)$ & $-0.583(74)$ \\
\hline$\Delta_{K}(\mathrm{kHz})$ & 0.1430 & 0.1699 & $1.02(41)$ & $4.4(18)$ \\
\hline$\delta_{J}(\mathrm{kHz})$ & 0.01605 & 0.01594 & $0.0271(49)$ & $\ldots$ \\
\hline$\delta_{K}(\mathrm{kHz})$ & $0.136(17)$ & $0.188(38)$ & $0.554(50)$ & $0.55(24)$ \\
\hline$F_{a b}(\mathrm{MHz})$ & $\pm 15.9976(45)$ & $\pm 15.9976(45)$ & $\pm 14.030(22)$ & $\pm 14.030(22)$ \\
\hline$\Delta_{0}(\mathrm{MHz})$ & $29380.7500(58)$ & $29380.7500(58)$ & $33744.2(22)$ & $33744.2(22)$ \\
\hline$a$-type assigned ${ }^{b}$ & 33 & 21 & 29 & 13 \\
\hline $0^{-} \leftarrow 0^{+}$assigned $^{\mathrm{b}}$ & 8 & 8 & 0 & 0 \\
\hline$\sigma(\mathrm{kHz})$ & 2.4 & 2.4 & 2.4 & 2.4 \\
\hline$\sigma(\mathrm{kHz})^{\mathrm{c}}$ & 687 & 687 & 234 & 234 \\
\hline
\end{tabular}

${ }^{2}$ Uncertainties are type A, $k=1$, or $1 \sigma$ (Ref. 29).

${ }^{\mathrm{b}}$ Experimental uncertainty of all assigned lines is $4 \mathrm{kHz}$.

' Observed minus calculated standard deviation of fits that exclude $F_{a b}, \Delta_{0}$ and the $0^{-} \leftarrow 0^{+}$transitions. 
TABLE IV. $S_{0}$ rotational constants of the asymmetric ${ }^{13} \mathrm{C}$ and ${ }^{18} \mathrm{O}$ isotopomers of tropolone obtained from fits of the FTMW spectra.

\begin{tabular}{|c|c|c|c|}
\hline & \multicolumn{2}{|c|}{${ }^{13} \mathrm{C}_{2(1)}$} & \multirow{2}{*}{$\frac{{ }^{18} \mathrm{O}}{0^{+a}}$} \\
\hline & $0^{+}$ & $0^{-\mathrm{a}}$ & \\
\hline$A(\mathrm{MHz})$ & $2734.7514(34)^{b}$ & $2735.5918(66)$ & $2702.2798(58)$ \\
\hline$B(\mathrm{MHz})$ & $1656.26912(73)$ & $1656.4474(18)$ & $1616.7946(71)$ \\
\hline$C(\mathrm{MHz})$ & $1031.82535(34)$ & $1032.0261(37)$ & $1011.84586(56)$ \\
\hline$\Delta I\left(\mathrm{u} \AA^{2}\right)$ & -0.13863 & -0.14429 & -0.13789 \\
\hline$\Delta_{J}(\mathrm{kHz})$ & $0.0913(85)$ & $0.141(59)$ & $0.0916(94)$ \\
\hline$\Delta_{J K}(\mathrm{kHz})$ & $2.996(30)$ & $-4.140(41)$ & $2.72(11)$ \\
\hline$\Delta_{K}(\mathrm{kHz})$ & $-1.57(45)$ & $7.61(72)$ & $-1.12(72)$ \\
\hline$\delta_{J}(\mathrm{kHz})$ & $0.0366(46)$ & $0.026(13)$ & $0.0377(48)$ \\
\hline$\delta_{K}(\mathrm{kHz})$ & $2.139(42)$ & $-0.714(95)$ & $2.17(70)$ \\
\hline$F_{a b}(\mathrm{MHz})$ & $\pm 1.226(37)$ & $\pm 1.226(37)$ & $\ldots$ \\
\hline$\Delta_{0}(\mathrm{MHz})$ & $26819(91)$ & $26819(91)$ & $\cdots$ \\
\hline Assigned $^{c}$ & 27 & 13 & 38 \\
\hline $0^{-} \leftarrow 0^{+}$assigned $^{\mathrm{c}}$ & 0 & 0 & 0 \\
\hline$\sigma(\mathrm{kHz})$ & 2.5 & 2.5 & 2.2 \\
\hline$\sigma(\mathrm{kHz})^{\mathrm{d}}$ & $11.2^{\mathrm{e}}$ & $11.2^{\mathrm{e}}$ & $\ldots$ \\
\hline
\end{tabular}

${ }^{\mathrm{a} A d d i t i o n a l ~ t e r m s ~ u s e d ~} \mathrm{C}_{2(1)} / 0^{-} H_{J}=1.07(57) \mathrm{Hz}$ and $\mathrm{O} / 0^{+} H_{J K K}$ $=18.3(70) \mathrm{Hz}$.

${ }^{\mathrm{b}}$ Uncertainties are type A, $k=1$, or $1 \sigma$ (Ref. 29).

${ }^{c}$ Experimental uncertainty of all assigned lines is $4 \mathrm{kHz}$.

${ }^{\mathrm{d}}$ Observed minus calculated standard deviation of fits that exclude $F_{a b}$ and $\Delta_{0}$.

${ }^{\mathrm{e}}$ Same $\sigma$ is obtained when $0^{+}$and $0^{-}$states are reversed in the fits that include $F_{a b}$ and $\Delta_{0}$.

level system. For $J=1$, the operator $F_{a b}\left(P_{b} P_{a}+P_{a} P_{b}\right)$ couples the two asymmetric rotor wave functions $|111\rangle$ and $|101\rangle\left(\left|J K_{a} K_{c}\right\rangle\right)$ of the $0^{+}$and $0^{-}$tunneling states. The Hamiltonian matrix of one of these pairs has the standard form

$$
\hat{H} \equiv\left[\begin{array}{cc}
E_{|101\rangle}^{(+)} & F_{a b} \\
F_{a b} & E_{|111\rangle}^{(-)}+\Delta_{0}
\end{array}\right] .
$$

Analytical solution of the corresponding secular equation of Eq. (1) yields the following eigenvalues:

$E^{( \pm)}=\frac{E_{|101\rangle}^{(+)}+E_{|111\rangle}^{(-)}+\Delta_{0} \mp \sqrt{\left(E_{|111\rangle}^{(-)}+\Delta_{0}-E_{|101\rangle}^{(+)}\right)^{2}+4 F_{a b}^{2}}}{2}$.

In the absence of tunneling-rotation interactions (i.e., $F_{a b}=0$ ), the eigenvalues are simply those of the asymmetric rotor with the $0^{-}$state offset by the vibrational energy difference. However, depending on the measurement precision and their relative magnitudes, $F_{a b}$ and $\Delta_{0}$ may be reliably obtained from the pure rotational transitions alone. For example, the asymmetric rotor levels in the $S_{0}$ state of tropolone are shifted by -9 and $+8 \mathrm{kHz}$ for the $|0,101\rangle$ and $|1,111\rangle\left(\left|v, J K_{a} K_{c}\right\rangle\right)$ levels, respectively. Of course, when the much weaker level-crossing transitions are observed, the value of $\Delta_{0}$ is determined directly and significantly decreases the correlation of these two parameters. To illustrate the impact of these terms, the observed-minus-calculated $(\mathrm{OmC})$ standard deviations from fits without them are given in the lower parts of the Tables I-IV. The overall fit quality de- creases by roughly an order of magnitude or more even for the two cases where the level-crossing transitions were not observed.

As a final note, the sign of the parameter $F_{a b}$ does not affect the rotational energies and consequently, is not determined from the frequency fits. However, the operator $P_{b} P_{a}$ $+P_{a} P_{b}$ is the same as that used to model rotational transition intensity perturbations arising from inertial axis reorientation effects. ${ }^{30}$ While this operator is a necessary condition for observing quantum interference effects, ${ }^{31}$ it is not sufficient for tropolone because of the additional requirement of hybrid band character. For the effective $C_{2 \nu}$ structure of tropolone on the time scale of a rotational period, the dipole moments lie along the $a$ inertial axes, and consequently, the transition intensities will be insensitive to the sign of $F_{a b}$. The $b$-axis components present in the asymmetric structures are too small to make appreciable intensity differences in the MW spectra. These same arguments apply to the electronically excited states of tropolone discussed in the next section.

\section{B. $S_{1}$ state of tropolone}

The two lowest-energy features in the $S_{1}$ spectrum of tropolone have been assigned by Alves and Hollas to the $0^{+} \leftarrow 0^{+}$and $0^{-} \leftarrow 0^{-}$transitions at 27017.54 and $27036.47 \mathrm{~cm}^{-1}$, respectively. ${ }^{13,14}$ The rotationally resolved UV spectra of these bands are shown in Fig. 3. Each spectrum spans more than a $7 \mathrm{~cm}^{-1}$ spectral region at a rotational temperature of $\approx 9 \mathrm{~K}$. As apparent from the absence of $a$-type $Q$-branch transitions near the electronic origins, the rotational spectra of $0^{+} \leftarrow 0^{+}$and $0^{-} \leftarrow 0^{-}$bands are $100 \% b$ type in character. The band polarizations are consistent with the $C_{2 \nu}$ symmetry of the transition state and the $B_{2}$ electronic symmetry of the $S_{1}$ state. A number of $a$-type transitions are also observed for the much weaker $0^{+} \leftarrow 0^{-}$and $0^{-} \leftarrow 0^{+}$vibronic bands. These transitions are shifted to the red and blue of the $0^{+} \leftarrow 0^{+}$and $0^{-} \leftarrow 0^{-}$bands, respectively, as a result of the much smaller $\approx 1 \mathrm{~cm}^{-1}$ ground-state splitting and therefore, are nested within these main bands. Nominally forbidden because of parity selection rules, the appearance of the $a$-type transitions is a result of the mixed nature of the rovibrational wave functions from tunneling-rotation interactions between the $0^{+}$and $0^{-}$levels of both electronic states. Rovibronic transitions of this latter kind are $a$ type in character because of the $B_{2}$ symmetry of the $0^{-}$levels. The relative contributions of the $0^{+} \leftarrow 0^{-}$and $0^{+} \leftarrow 0^{+}$bands are shown in Fig. 4.

The assigned line sets of the $0^{+} \leftarrow 0^{+}$and $0^{-} \leftarrow 0^{-}$bands were simultaneously fitted using the SPFIT program. ${ }^{28}$ To aid in the assignment and fitting of these spectra, the predicted line sets generated by SPFIT were overlayed with the experimental data using the JB95 program. ${ }^{32}$ The assigned line sets included 129 and 102 transitions from the $0^{+} \leftarrow 0^{-} / 0^{+} \leftarrow 0^{+}$ and $0^{-} \leftarrow 0^{+} / 0^{-} \leftarrow 0^{-}$bands, respectively. The assigned frequencies were then used to refine the relative frequency calibration of the UV spectra by means of a lower-state combination difference analysis. ${ }^{25}$ All fits discussed below include the pure rotational transitions of the $S_{0}$ state weighted in 


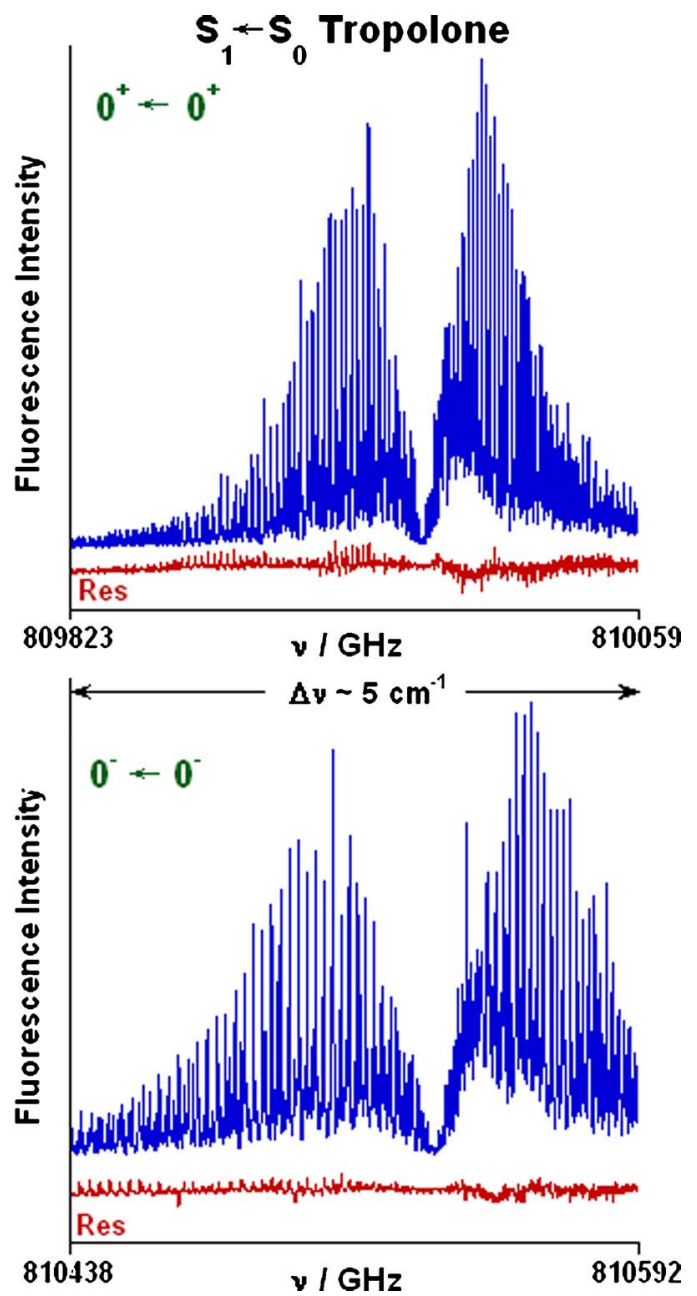

FIG. 3. Rotationally resolved UV spectra of the $0^{+} \leftarrow 0^{+}\left(0^{+} \leftarrow 0^{-}\right)$and $0^{-}$ $\leftarrow 0^{-}\left(0^{-} \leftarrow 0^{+}\right)$transitions at $\lambda=370.128$ and $369.869 \mathrm{~nm}$, respectively. Both bands span more than $5 \mathrm{~cm}^{-1}$ at rotational temperatures of $\sim 10 \mathrm{~K}$. Residuals are shown to illustrate the quality of the frequency and intensity fits.

proportion to the measurement uncertainties of $2 \mathrm{kHz}$ and 5 $\mathrm{MHz}$ for the MW and UV data, respectively.

The parameters used for the initial fits of the $S_{1}$ states included the six rotational constants and the two band origins. The quartic distortion parameters were not reliably fit-

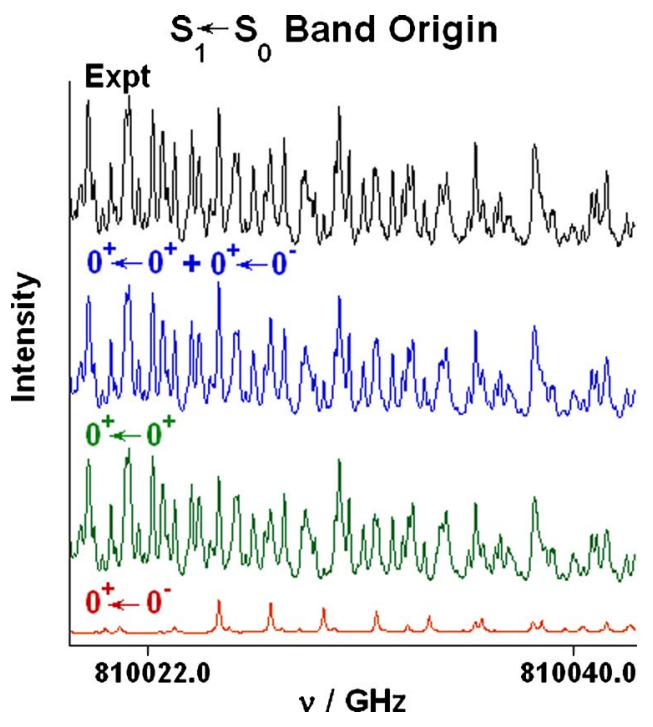

FIG. 4. Expanded region near the electronic origin illustrating contributions from the $0^{+} \leftarrow 0^{+}$and $0^{+} \leftarrow 0^{-}$vibrational transitions.

ted for either state. The OmC standard deviation of the overall fit was $5.5 \mathrm{MHz}$ which is $<1 / 20$ of the observed linewidths. Fits that included the tunneling-rotation term gave $F\left(S_{1}\right)=-6.9(9) \mathrm{MHz}$ and reduced the standard deviation only marginally to $5.4 \mathrm{MHz}$. The analysis was repeated using the ten distortion constants and $F\left(S_{1}\right)=$ $-4.28(24) \mathrm{MHz}$ previously reported for the $S_{1}$ state. ${ }^{15}$ The OmC standard deviation increased slightly to $6.8 \mathrm{MHz}$. Upon including all of the $S_{1}$ parameters in Ref. 15, the OmC standard deviation rose to $65 \mathrm{MHz}$. Except for the latter case, the changes in the overall fit quality are not statistically meaningful for our data sets. Therefore, the parameters reported in Table $\mathrm{V}$ are based on the initial fits that exclude $F\left(S_{1}\right)$ and the distortion constants. We also note that changes in the rotational constants for fixed values of $\left|F\left(S_{1}\right)\right|$ $<10 \mathrm{MHz}$ are less than the reported uncertainties in the constants.

Additional fits of the LIF intensities were performed using genetic algorithms ${ }^{33}(\mathrm{GA})$ and nonlinear least-squaresfitting routines based on the Levenberg-Marquardt

TABLE V. $S_{1}$ rotational constants of the $0^{+}$and $0^{-}$states of tropolone obtained from weighted least-squares fits with the ground-state data (see Table I for parameters). The Lorentzian linewidths and rotational temperatures were obtained from nonlinear least-squares fits of the observed band intensities.

\begin{tabular}{ccc}
\hline \hline & $0^{+}$ & $0^{-}$ \\
\hline$A / \Delta A(\mathrm{MHz})$ & $2690.8348(52) /-52.2505(52)^{\mathrm{a}}$ & $2686.1727(99) /-56.5387(99)$ \\
$B / \Delta B(\mathrm{MHz})$ & $1653.893(11) /-5.998(11)$ & $1653.7344(86) /-6.1302(86)$ \\
$C / \Delta C(\mathrm{MHz})$ & $1025.7803(82) /-8.6032(82)$ & $1025.5307(10) /-8.7934(10)$ \\
$\Delta I(\mathrm{u} \AA)^{2}$ & -0.70669 & -0.94203 \\
Origin $\left(\mathrm{cm}^{-1}\right)$ & $27017.67(2)$ & $27036.58(2)$ \\
Band type & $100 \% b$ type & $100 \% b$ type \\
$\Delta v_{\text {Lor }}$ & $131.1(8)$ & $143.3(8)$ \\
$T_{1}: T_{2}:$ wt $^{\mathrm{K}}(\mathrm{K})^{\mathrm{b}}$ & $9.0(1): 33(1): 0.08(1)$ & $9.5(1): 46(1): 0.07(1)$ \\
Assigned & 129 & 102 \\
$\sigma\left(\mathrm{MHz}^{\mathrm{c}}\right.$ & 5.1 & 5.1 \\
\hline
\end{tabular}

\footnotetext{
Uncertainties are type A, $k=1$, or $1 \sigma$ (Ref. 29).

${ }^{\mathrm{b}}$ Two-temperature model from Ref. 35: $\exp \left(-\Delta E / k T_{1}\right)+\mathrm{wt} \exp \left(-\Delta E / k T_{2}\right)$.

${ }^{c}$ Experimental uncertainty of all assigned lines is $5 \mathrm{MHz}$.
} 
TABLE VI. Observed changes in the $S_{0}$ rotational constants, $\Delta A=A^{-}-A^{+}$, etc., of the two tunneling states of each isotopomer and the tunneling parameters relative to the parent isotopomer. All values are in $\mathrm{MHz}$ except where noted.

\begin{tabular}{ccccccc}
\hline \hline $0^{+}-0^{-}$ & ${ }^{12} \mathrm{C}$ & $\mathrm{OD}$ & ${ }^{13} \mathrm{C}_{5}$ & ${ }^{13} \mathrm{C}_{4(6)}$ & ${ }^{13} \mathrm{C}_{3(7)}$ & ${ }^{13} \mathrm{C}_{2(1)}$ \\
\hline$\Delta A$ & -0.37390 & $-0.010(3)$ & -0.3732 & -0.1766 & -0.7415 & +0.8404 \\
$\Delta B$ & -0.026750 & $-0.0019(5)$ & -0.02702 & -0.08181 & -0.1937 & +0.1783 \\
$\Delta C$ & -0.059435 & $-0.0050(3)$ & -0.05820 & -0.05239 & -0.1689 & +0.2007 \\
$\Delta \Delta I^{\mathrm{a}}$ & -1.42 & $+1.392(2)$ & -2.11 & -2.15 & -6.09 & -5.66 \\
& $\ldots$ & $\mathrm{OD}-{ }^{12} \mathrm{C}$ & ${ }^{13} \mathrm{C}_{5}{ }^{12} \mathrm{C}$ & ${ }^{13} \mathrm{C}_{4(6)}{ }^{12} \mathrm{C}$ & ${ }^{13} \mathrm{C}_{3(7)}{ }^{-12} \mathrm{C}$ & ${ }^{13} \mathrm{C}_{2(1)}-{ }^{12} \mathrm{C}$ \\
$\Delta F$ & $\cdots$ & $-7.632(2)$ & +0.56778 & +0.45927 & +2.427 & +15.231 \\
$\Delta \Delta_{0}$ & $\cdots$ & $-27670.570(5)$ & -114.7204 & +186.9531 & +4550 & -2375 \\
\hline \hline
\end{tabular}

${ }^{\mathrm{a}}$ Inertial defect in units of $\mathrm{u} \AA^{2} \times 10^{-3}$.

algorithm $^{34}$ to obtain the Lorentzian linewidths and the three additional parameters that define a two-temperature model (see footnote of Table V). ${ }^{35}$ The GA was first used to obtain global minima values for these parameters which were then fitted to convergence using the nonlinear least-squares algorithm. In both cases, the Gaussian component of the lineshape function was held fixed at the Doppler-limited resolution of 18(1) MHz. Nuclear-spin statistical weights were necessary to model the observed LIF intensities. The ratios used for $K_{a}$ even:odd were 10:6 for the $0^{+}$vibrational level and 6:10 for the $0^{-}$level. ${ }^{13,15}$ The parameters obtained from the nonlinear fits are also given in Table V. The residuals shown in Fig. 3 illustrate the quality of the intensity fits. The observed Lorentzian linewiths are 131.1(8) and 143.3(8) $\mathrm{MHz}$ for the $0^{+} \leftarrow 0^{-} / 0^{+} \leftarrow 0^{+}$and $0^{-} \leftarrow 0^{+} / 0^{-} \leftarrow 0^{-}$states, respectively. The increase of $12.2 \mathrm{MHz}$ is well outside of the $1 \sigma$ uncertainty of $\approx 1 \mathrm{MHz}$.

\section{DISCUSSION}

\section{A. $S_{0}$ tunneling dynamics in symmetric structures of tropolone}

Like the parent, the ${ }^{13} \mathrm{C}_{5}$ and $\mathrm{OD}$ isotopomers of tropolone have symmetric transition state structures and therefore, tunneling wave functions that are delocalized over the two symmetric wells of the potential-energy surface. Contributions from the two resonance forms I and II of Fig. 1 are therefore, equally weighted in each tunneling state. From Table VI, the $A$ rotational constants of the $0^{-}$state decrease relative to the $0^{+}$state, consistent with the nodal properties of the tunneling wave functions. The small negative inertial defects of the two tunneling states of the parent have been previously interpreted in terms of contributions from the two lowest-frequency out-of-plane vibrational modes of a planar frame. ${ }^{2,15}$ The similar values obtained for the OD and ${ }^{13} \mathrm{C}_{5}$ isotopomers (Table II) also support planar structures in these two tunneling states.

One striking feature of the data in Tables I and II is the inversion splitting $\Delta_{0}$ of $1523 \mathrm{MHz}$ for D-tropolone. This value is $1 / 19$ of the splitting for the hydrogen tunneling $(\approx 30000 \mathrm{MHz})$ and is in sharp contrast to the much smaller decrease observed for the ${ }^{13} \mathrm{C}_{5}$ isotopomer of only $0.4 \%$. Similar dependencies of the tunneling parameters on the symmetric isotopomers have been observed in malonaldehyde. ${ }^{36,37}$ In all cases, the decreases in the tunnel- ing splittings reflect kinetic isotope effects arising from increases in the reduced mass along the tunneling coordinates. Recent theoretical work on tropolone assumes a multidimensional proton-tunneling path that gives a reduced mass $\mu_{\mathrm{H}}$ for the inversion motion on the order of 6 or $7 \mathrm{amu}^{7}$ As discussed below, our measured ratio of 19 for the H/D isotopomer inversion splitting indicates that the ratio $\mu_{\mathrm{D}} / \mu_{\mathrm{H}}$ is approximately 2 , suggesting that $\mu_{\mathrm{H}}$ is $\approx 1$ amu.

Evidence for $\mu_{\mathrm{D}} / \mu_{\mathrm{H}} \approx 2$ comes from an estimate of the barrier height to inversion $V_{0}$ for a simple one-dimensional (linear) inversion path $s$ of length $2 s_{0}$ parallel to the $b$ axis of tropolone. For these calculations, the quartic-quadratic potential form was assumed,

$$
V(s)=V_{0}-2\left(V_{0} / s_{0}^{2}\right) s^{2}+\left(V_{0} / s_{0}^{4}\right) s^{4}
$$

having a barrier maximum $V_{0}$ at $s=0$ and energy minima at $s= \pm s_{0}$. We take $s_{0}$ to be the $r_{s} b$ coordinate of the mobile $\mathrm{D} / \mathrm{H}$ atom obtained using the Kraitchman equations. ${ }^{38} \mathrm{Di}-$ agonalization of the Hamiltonian that includes the potential surface of Eq. (3) gives a locus of values for $V_{0}$ and $\mu_{\mathrm{H}}$ that fit the experimental inversion frequency, $\Delta_{0}$ =29 193.7969 MHz. However, the Coriolis coupling constant $F_{a b}=16.4569(1) \mathrm{MHz}$ serves as an additional constraint and may be evaluated from the approximation given by Baughcum et al., ${ }^{37}$

$$
F_{a b}=\left(\mu a\left\langle 0^{+}|b| 0^{-}\right\rangle A B\right) /\left(505379 \text { amu A }{ }^{2} \mathrm{MHz}\right),
$$

where $a$ is the $r_{s} a$ coordinate of the $\mathrm{D} / \mathrm{H}$ atom, $\left\langle 0^{+}|b| 0^{-}\right\rangle$is the off-diagonal matrix element of the $b$ coordinate (from the fit), and $A$ and $B$ are the rotational constants of tropolone. The one point on the $V_{0} / \mu$ locus that fits both $\Delta_{0}$ and $F_{a b}$ gives $V_{0}=1883 \mathrm{~cm}^{-1}$ and $\mu_{\mathrm{H}}=1.3903 \mathrm{amu}$. Similar calculations on OD and ${ }^{13} \mathrm{C}_{5}$ give $2288 \mathrm{~cm}^{-1}$ and $2.0439 \mathrm{amu}$ and $1929 \mathrm{~cm}^{-1}$ and $1.3653 \mathrm{amu}$ for $V_{0}$ and $\mu$, respectively. The results for the two limiting cases are shown graphically in Fig. 5. The discrepancy of $405 \mathrm{~cm}^{-1}$ for $V_{0}$ is a measure of the reliability of this simple tunneling model for tropolone. We note, however, that this range of values is consistent with more sophisticated theoretical models that predict a barrier of $2161 \mathrm{~cm}^{-1}$ and tunneling splittings of 3.5 and $0.16 \mathrm{~cm}^{-1}$ for the $\mathrm{OH}$ and $\mathrm{OD}$ isotopomers, respectively. ${ }^{11}$ 
Values of $V_{0}$ and $\mu$ which fit the inversion splitting of $\mathrm{H}$-tropolone and $\mathrm{D}$-tropolone

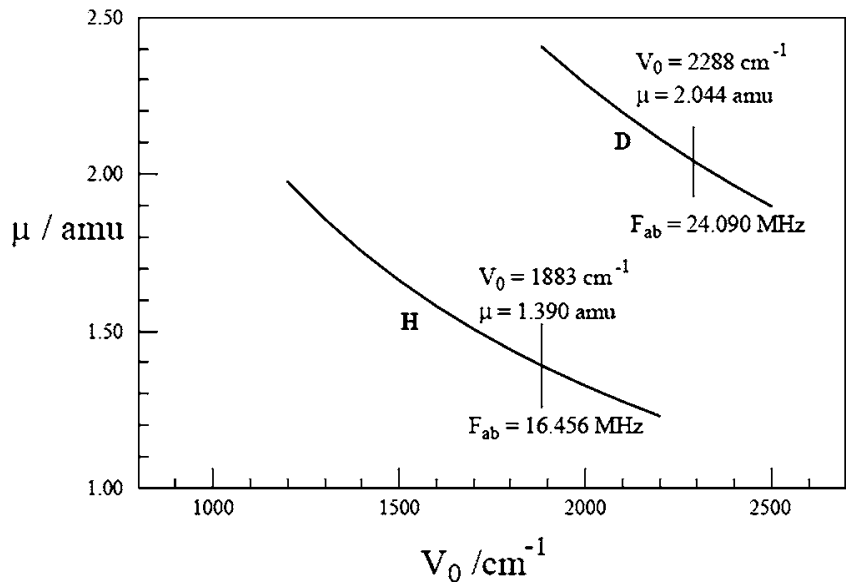

FIG. 5. The two curves represent the locus of $V_{0} / \mu$ pairs that give the experimental values of the inversion splittings for $\mathrm{H}$ - and $\mathrm{D}$-tropolone. The model on which these curves are based is given in the text. However, the various points on the curves give varying values for the Coriolis coupling constants $F_{a b}$. The vertical lines represent the points on the curves where the experimental values of $F_{a b}$ are predicted. Those two points, one for each curve, are the values of $V_{0}$ and $\mu$ which correctly predict the experimental values of the inversion splittings and the Coriolis coupling constants for the two isotopomers. Ideally, the two predicted values for $V_{0}$ should be identical for both $\mathrm{H}$ - and D-tropolone. The difference of $405 \mathrm{~cm}^{-1}$ between the two values of $V_{0}$ is an indication of the limitations of the simple one-dimensional linear inversion model.

\section{B. Impact of asymmetry on the tunneling dynamics in $S_{0}$}

For the remaining isotopomers, the asymmetric substitutions reduce the $C_{2 v}$ symmetry of the transition state and may partially or completely quench the tunneling dynamics. ${ }^{36} \mathrm{De}$ pending on the extent of the perturbation, the $0^{+}$and $0^{-}$wave functions will become increasingly localized in one of the two wells and therefore, begin to favor one of the two resonance forms. In line with the expected magnitude of the quenching, the data sets in Tables III and IV are arranged in order of decreasing distance of the substituted atom to the tunneling proton. The changes in the rotational constants and tunneling parameters relative to those of the parent isotopomer are summarized in Table VI.

The ${ }^{13} \mathrm{C}_{4(6)}$ isotopomer appears to retain much of the character of the symmetric structures. However, the tunneling splitting increases from that of the ${ }^{12} \mathrm{C}$ parent by $>0.5 \%$, offsetting the expected decrease from the kinetic isotope effect on symmetric forms. The perturbations of the remaining three isotopomers become increasingly more severe for positions approaching the tunneling proton. The tunneling splitting increases by $15 \%$ and then decreases by $8 \%$ for the ${ }^{13} \mathrm{C}_{3(7)}$ and ${ }^{13} \mathrm{C}_{2(1)}$ isotopomers, respectively. The magnitude of $F_{a b}$ decreases by $15 \%$ for the ${ }^{13} \mathrm{C}_{3(7)}$ and then by more than ten fold for ${ }^{13} \mathrm{C}_{2(1)}$. The Coriolis interactions are completely absent for the ${ }^{18} \mathrm{O}$ isotopomer suggesting that the tunneling is completely quenched and that the $0^{+}$and $0^{-}$ wave functions are highly localized. Finally, notice that in sharp contrast to the other isotopomers (including the parent), the three rotational constants of ${ }^{13} \mathrm{C}_{2(1)}$ increase in the $0^{-}$state.

The anomalous changes in the rotational constants and tunneling splitting for the ${ }^{13} \mathrm{C}_{2(1)}$ isotopomer are two of the more intriguing aspects of tropolone's tunneling dynamics. One explanation might be that the state labels were reversed in the fit. This, however, proved not to be the case. The OmC standard deviation from such fits increased to $11 \mathrm{kHz}$, a result identical to fits that excluded $F_{a b}$ and $\Delta_{0}$. A second explanation might involve a reversal of the localized probability amplitudes of the $0^{+}$and $0^{-}$wave functions relative to those of the other asymmetrically substituted atoms.

In order to explore this second possibility further, the in-plane center-of-mass (COM) coordinates of the substituted atoms were determined using Kraitchman's equations ${ }^{38}$ and are given for each state and each isotopomer in

TABLE VII. Experimentally derived in-plane substitution coordinates of the heavy atoms in tropolone. Planar structures are assumed. Theoretical values were obtained at the MP2/aug-cc-pVTZ level of theory for resonance structure I of Fig. 1.

\begin{tabular}{|c|c|c|c|c|c|}
\hline & & \multicolumn{2}{|c|}{ Experimental $^{\mathrm{a}}$} & \multicolumn{2}{|c|}{ Theory } \\
\hline & & $0^{+}$ & $0^{-}$ & $\mathrm{C}-\mathrm{OH}$ & $\mathrm{C}=\mathrm{O}$ \\
\hline \multirow[t]{2}{*}{$\mathrm{C}_{5}$} & $a(\AA)$ & $-2.3059(6)$ & $-2.3060(6)$ & -2.304 & -2.304 \\
\hline & $b(\AA)$ & $0^{\mathrm{b}}$ & $0^{\mathrm{b}}$ & -0.021 & -0.021 \\
\hline \multirow{2}{*}{$\mathrm{C}_{4(6)}$} & $a(\AA)$ & $-1.6908(9)$ & $-1.6902(9)$ & -1.690 & -1.690 \\
\hline & $b(\AA)$ & $-1.260(1)$ & $+1.249(1)$ & -1.259 & +1.243 \\
\hline \multirow[t]{2}{*}{$\mathrm{C}_{3(7)}$} & $a(\AA)$ & $-0.311(5)$ & $-0.350(4)$ & -0.323 & -0.355 \\
\hline & $b(\AA)$ & $-1.575(1)$ & $+1.583(1)$ & -1.564 & +1.581 \\
\hline \multirow[t]{2}{*}{$\mathrm{C}_{2(1)}$} & $a(\AA)$ & $+0.806(2)$ & $+0.780(2)$ & +0.761 & +0.813 \\
\hline & $b(\AA)$ & $+0.753(2)$ & $-0.696(2)$ & -0.708 & +0.757 \\
\hline \multirow[t]{2}{*}{$\mathrm{O}_{8(9)}$} & $a(\AA)$ & $+2.0032(7)$ & $\cdots$ & +1.983 & +1.971 \\
\hline & $b(\AA)$ & $+1.227(1)$ & $\cdots$ & -1.238 & +1.246 \\
\hline
\end{tabular}

${ }^{a}$ Signs assigned to the experimentally derived coordinates are included to clarify associations made in the text. Uncertainties are type B and $k=1$ (Ref. 29) based on Costain's criteria from Ref. 39.

${ }^{\mathrm{b}}$ Small imaginary coordinate obtained. 
TABLE VIII. Calculated differences in the harmonic zero-point energies of the heavy-atom isotopomers of tropolone relative to the ${ }^{12} \mathrm{C}$ parent at the MP2/6-311++G(d,p) level. Also given are the calculated ZPE differences between members of the asymmetric pairs and those determined from the two-state model given in Eq. (5). The observed tunneling splittings are given in the last column. All values are in $\mathrm{cm}^{-1}$.

\begin{tabular}{|c|c|c|c|c|c|c|}
\hline \multicolumn{5}{|c|}{$a b$ initio theory } & \multirow{2}{*}{$\frac{\text { Model }}{\Delta_{\mathrm{ZPE}}^{\mathrm{c}}}$} & \multirow{2}{*}{$\frac{\text { Expt. }}{\Delta_{0}^{\mathrm{d}}}$} \\
\hline $\mathrm{C}-\mathrm{OH}$ & ${ }^{13-12} \Delta_{\mathrm{ZPE}}{ }^{\mathrm{a}}$ & $\mathrm{C}=\mathrm{O}$ & ${ }^{13-12} \Delta_{\mathrm{ZPE}}{ }^{\mathrm{a}}$ & ${ }^{\mathrm{L}-\mathrm{R}} \Delta_{\mathrm{ZPE}}{ }^{\mathrm{b}}$ & & \\
\hline${ }^{13} \mathrm{C}_{5}$ & -45.64 & ${ }^{13} \mathrm{C}_{5}$ & -45.64 & 0.00 & 0.00 & 0.97 \\
\hline${ }^{13} \mathrm{C}_{4}$ & -46.27 & ${ }^{13} \mathrm{C}_{6}$ & -45.36 & -0.91 & 0.14 & 0.98 \\
\hline${ }^{13} \mathrm{C}_{3}$ & -46.39 & ${ }^{13} \mathrm{C}_{7}$ & -44.81 & -1.58 & 0.57 & 1.13 \\
\hline${ }^{13} \mathrm{C}_{2}$ & -50.92 & ${ }^{13} \mathrm{C}_{1}$ & -52.31 & +1.39 & $\mathrm{e}$ & 0.89 \\
\hline${ }^{18} \mathrm{O}_{8}$ & -40.22 & ${ }^{18} \mathrm{O}_{9}$ & -37.65 & -2.57 & $\mathrm{f}$ & $\mathrm{f}$ \\
\hline \multicolumn{7}{|c|}{ 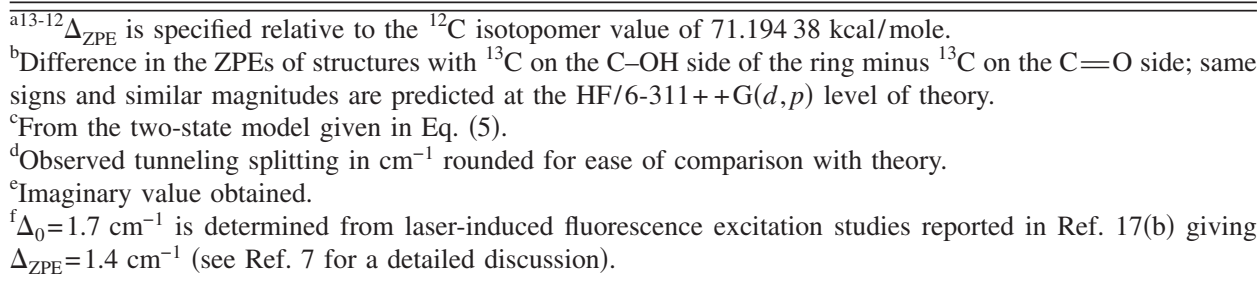 } \\
\hline
\end{tabular}

Table VII. These are compared with the atomic coordinates predicted for structure I in Fig. 1 at the MP2/aug-cc-pVTZ level of theory. Notice first that these coordinates unambiguously confirm the semiquantitative arguments given above to identify each isotopomer. The predicted coordinates of ${ }^{13} \mathrm{C}_{5}$ and $\mathrm{C}_{4(6)}$ are in excellent agreement with experiment. For the $\mathrm{C}_{4(6)}$ structure, the experimental $b$ coordinates differ, however, by $0.011 \AA$ versus the difference of $0.016 \AA$ found theoretically. This would suggest that ${ }^{13} \mathrm{C}_{4}$ substitution causes the $0^{+}$wave function to become somewhat localized on the negative $b$ axis or $\mathrm{C}-\mathrm{OH}$ side of the ring and the $0^{-}$ wave function on the $\mathrm{C}=\mathrm{O}$ side. A comparison of the $\mathrm{C}_{3(7)}$ coordinates reveals a similar association with tunneling state.

This trend changes abruptly for the $\mathrm{C}_{2(1)}$ coordinates. The coordinate differences in $a$ and $b$ are somewhat larger; 0.026 and $0.057 \AA$ versus predicted values of 0.052 and $0.049 \AA$, respectively. More importantly, their absolute magnitudes suggest that the probability amplitudes of the $0^{+}$and $0^{-}$states are reversed, i.e., the ${ }^{13} \mathrm{C}_{1}$ atom on the $\mathrm{C}=\mathrm{O}$ side is more favored in the lower-energy $0^{+}$state and ${ }^{13} \mathrm{C}_{2}$ on the $\mathrm{C}-\mathrm{OH}$ side is the preferred structure in the $0^{-}$state.

\section{Isotope driven dynamics in the zero-point energy levels of $S_{0}$}

Within the Born-Oppenheimer approximation, the structures favored by the partially or completely localized wave functions of each asymmetric pair are determined by the relative magnitudes of their zero-point energies (ZPEs). The ZPE of each isotopomer includes contributions from 39 vibrational degrees of freedom. The heavier isotopes will have larger reduced masses and therefore, diminished ZPE contributions along one or more of these normal-mode coordinates. 40,41

Insight into the magnitude of the asymmetry and its impact on the tunneling dynamics may be obtained from a simple two-state model. Since the ZPE difference for each ${ }^{13} \mathrm{C}$ pair introduces, in effect, an asymmetry to the tunneling potential, the perturbations may be treated by adding a term to the diagonal elements of the symmetric two-state Hamiltonian matrix [very similar in form to that given in Eq. (1)]. ${ }^{7,42}$ As shown previously, ${ }^{7}$ the eigenvalues are related to the experimental tunneling splitting $\Delta_{\mathrm{AS}}$ using

$$
\Delta_{\mathrm{AS}}^{2}=\Delta_{S}^{2}+\Delta_{\mathrm{ZPE}}^{2},
$$

where $\Delta_{S}$ is the splitting observed for the symmetric form and $\Delta_{\mathrm{ZPE}}$ is the ZPE difference of a given asymmetric pair. Furthermore, the probability of tunneling into the higher energy well is obtained from $P_{\mathrm{rel}}=\left[1+\Delta_{\mathrm{ZPE}}^{2} / \Delta_{S}^{2}\right]^{-1}$. The results given in the fourth column of Table VIII illustrate that the $\Delta_{\text {ZPE }}$ accounts for only $1 / 5$ of the observed splitting for the ${ }^{13} \mathrm{C}_{4(6)}$ pair, and therefore, the probability density of the wave function in each well is nearly equal with $P_{\text {rel }}=98 \%$. For the ${ }^{13} C_{3(7)}$ pair, $\Delta_{\text {ZPE }}$ is more important, accounting for roughly half of the observed splitting and leading to increased wave-function asymmetry where now $P_{\text {rel }}=74 \%$. However, the two-state model fails for $\mathrm{C}_{2(1)}$ asymmetric pair since the $\Delta_{\mathrm{ZPE}}$ can never be less than $\Delta_{S}$. The off-diagonal terms in the Hamiltonian matrix that describe the tunneling interaction are assumed to remain unaltered in this model. The greater than 10 fold reduction in $F_{a b}$ observed for the ${ }^{13} \mathrm{C}_{2(1)}$ isotopomer versus the other ${ }^{13} \mathrm{C}$ isotopomers (see Tables II-IV) indicates that this assumption is not valid and that the magnitudes of the off-diagonal terms must also decrease. In the event of an equivalent 13-fold reduction in the off-diagonal term $\Delta_{S} / 2, \Delta_{\mathrm{ZPE}}$ would account for $99 \%$ of the observed splitting.

Estimates of the $\Delta_{\mathrm{ZPE}}$ may also be obtained from $a b$ initio theory. The calculated decreases in the zero-point energies relative to the parent are given in Table VIII for the nine heavy isotopomers of reference structure I. These are the harmonic values obtained at the MP2/6311++ $\mathrm{G}(d, p)$ level using tight convergence criteria. ${ }^{43}$ All values are negative for the heavier isotopes (as expected from the product rule ${ }^{40}$ ) and range between -37 and $-53 \mathrm{~cm}^{-1}$. Of particular interest are the changes within each asymmetric pair given in the third column. The negative $\Delta_{\mathrm{ZPE}}$ 's of the asymmetric 
TABLE IX. Range of values that are possible for the radiative decay rates $k_{r}$, nonradiative rates $k_{\mathrm{n}}$, and quantum yields $\varphi$ based on the measured lifetimes and relative fluorescence quantum yields (Ref. 45) of tropolone and tropolone- $\mathrm{H}_{2} \mathrm{O}$ and Eq. (7).

\begin{tabular}{|c|c|c|c|c|c|c|c|c|c|}
\hline \multicolumn{7}{|c|}{ Tropolone decay rate $\left(10^{8} \mathrm{~s}^{-1}\right)$} & \multicolumn{3}{|c|}{ Tropolone- $\mathrm{H}_{2} \mathrm{O}$ decay rate $\left(10^{8} \mathrm{~s}^{-1}\right)$} \\
\hline$k_{r}\left(0^{+}\right)$ & $k_{\mathrm{nr}}\left(0^{+}\right)$ & $\varphi\left(0^{+}\right)$ & $k_{r}\left(0^{-}\right)$ & $k_{\mathrm{nr}}\left(0^{-}\right)$ & $\varphi\left(0^{-}\right)$ & $k_{\mathrm{nr}}\left(0^{-}\right) / k_{\mathrm{nr}}\left(0^{+}\right)$ & $k_{r}$ & $k_{\mathrm{nr}}$ & $\varphi$ \\
\hline 0.00 & 8.23 & 0.00 & 0.00 & 9.00 & 0.00 & 1.09 & 0.00 & 4.26 & 0.00 \\
\hline $0.50^{\mathrm{a}}$ & 7.73 & 0.06 & 0.49 & 8.51 & 0.05 & 1.10 & 2.05 & 2.22 & 0.48 \\
\hline 1.04 & 7.19 & 0.12 & 1.00 & 8.00 & 0.11 & 1.11 & 4.26 & 0.00 & $1.00^{\mathrm{b}}$ \\
\hline
\end{tabular}

${ }^{\mathrm{a}}$ Radiative rate estimated for tropolone in cyclohexane.

${ }^{\mathrm{b}}$ Establishes upper bounds on $k_{r}\left(0^{+}\right)$and $k_{r}\left(0^{-}\right)$.

pairs ${ }^{13} \mathrm{C}_{4} /{ }^{13} \mathrm{C}_{6},{ }^{13} \mathrm{C}_{3} /{ }^{13} \mathrm{C}_{7}$, and ${ }^{18} \mathrm{O}_{8} /{ }^{18} \mathrm{O}_{9}$ indicate that the lower-energy $0^{+}$wave function is preferentially localized on the $\mathrm{C}-\mathrm{OH}$ side of the ring and the $0^{-}$wave function on the $\mathrm{C}=\mathrm{O}$ side. In contrast, this association is reversed for the ${ }^{13} \mathrm{C}_{2} /{ }^{13} \mathrm{C}_{1}$ pair, indicating also a side reversal of the probability densities of the wave functions. These conclusions are entirely consistent with those based on the substituted atom coordinates. Although the $\Delta_{\mathrm{ZPE}}$ 's are small $(<0.01 \%$ of the total ZPE), it is clear that subtle perturbations within the frame of tropolone can have a remarkable impact on the average location of the proton in the two tunneling states of tropolone.

\section{Nonradiative decay dynamics of tropolone in the $S_{1}$ state}

Many of the photophysical properties of the $S_{1}$ state of tropolone have been previously interpreted from vibronically resolved measurements performed in jet-cooled expansions ${ }^{17,19,44,45}$ and from high-resolution four-wave mixing data obtained at room temperature. ${ }^{15}$ The important $S_{1}$ property uncovered in the rotationally resolved studies is directly relevant to the nonradiative decay dynamics of the $S_{1}$ state. We find all of the rotational lines in both tunneling states to be smooth Lorentzian shapes (after deconvolution of the small $18 \mathrm{MHz}$ Gaussian component arising from residual Doppler broadening). Furthermore, the Lorentzian widths are different for the two states but constant across each band and therefore, lack any $J$ or $K_{a}$ dependence for $J=0-22$ and $K_{a}=0-22$ sampled at $\approx 9 \mathrm{~K}$. The fluorescence decay rates $k_{F}$ that correspond to the observed widths of 131.1(8) and 143.3(8) $\mathrm{MHz}$ for the $0^{+}$and $0^{-}$levels are $8.23(1) \times 10^{8}$ and $9.00(1) \times 10^{8} \mathrm{~s}^{-1}$, respectively. The rate increase of $0.77(2) \times 10^{8} \mathrm{~s}^{-1}$ over the $\approx 20 \mathrm{~cm}^{-1}$ energy interval is slightly larger than that previously obtained from fluorescence lifetime measurements. ${ }^{44}$

The relative changes in a related quantity $\varphi_{F}$, the fluorescence quantum yield, have been measured by Hagemeister et al. ${ }^{45}$ for the $0^{+}$and $0^{-}$states and other vibronic levels in $S_{1}$ up to $\Delta E \approx+650 \mathrm{~cm}^{-1}$ above the origin using cavity ring down methods. The quantum yield $\varphi_{F}=k_{r} /\left(k_{r}+k_{\mathrm{nr}}\right)$ is defined as the ratio of radiative decay rate $k_{r}$ to the fluorescence decay rate $k_{F}$, which includes contributions from both $k_{r}$ and the nonradiative rate(s) $k_{\mathrm{nr}}$. The relative quantum yield reported for the $0^{+}$and $0^{-}$levels is $\varphi\left(0^{-}\right) / \varphi\left(0^{+}\right)=0.89(6)$ which together with the $k_{F}$ values measured here determine the ratio $k_{r}\left(0^{-}\right) / k_{r}\left(0^{+}\right)=0.97(6)$.
The absolute quantum yield has not been measured for gas-phase tropolone and therefore, $\mathrm{k}_{n r}$ is not known. However, the results from two other studies of the tropolonewater complex (TW) permit rigorous limits to be set on $k_{\mathrm{nr}}$ (and $k_{r}$ ) in tropolone. The quantum yield of TW obtained from the cavity ring down measurements ${ }^{45}$ is reported to undergo eight-fold increase relative to the tropolone origin, i.e., $\varphi(\mathrm{TW}) / \varphi\left(0^{+}\right)=8(1)$. Rotationally resolved measurements of the $S_{1}$ origin of TW have also been performed and will be reported elsewhere. From the line-shape fits of TW, the Lorentzian width obtained is $68(1) \mathrm{MHz}$, yielding a fluorescence decay rate $k_{F}(\mathrm{TW})$ of $4.27(6) \times 10^{8} \mathrm{~s}^{-1}$. Substituting the known quantities into the quantum yield ratio

$$
\frac{\phi(\mathrm{TW})}{\phi\left(0^{+}\right)}=\frac{k_{r}(\mathrm{TW})}{k_{r}\left(0^{+}\right)} \frac{k_{F}\left(0^{+}\right)}{k_{F}(\mathrm{TW})}
$$

reveals that the eight-fold larger quantum yield for TW is principally a result of the radiative rate increase where $k_{r}(\mathrm{TW}) / k_{r}\left(0^{+}\right)=4.1(6)$, with the remainder of the increase appearing in $k_{F}\left(0^{+}\right) / k_{F}(\mathrm{TW})=1.93(3)$. Under these constraints, decay rates relevant to tropolone may be obtained upon expanding and rearranging the ratio $k_{F}\left(0^{+}\right) / k_{F}(\mathrm{TW})$ to give

$$
k_{\mathrm{nr}}(\mathrm{TW})=\frac{8.23(1) \times 10^{8} \mathrm{~s}^{-1}}{1.93(3)}-4.1(6) k_{r}\left(0^{+}\right) \text {. }
$$

While this expression does not uniquely determine $k_{r}\left(0^{+}\right)$[and therefore, $k_{\mathrm{nr}}\left(0^{+}\right)$], it provides for a rather restrictive range on the possible values. The few important limiting cases are tabulated in Table IX. For example, in the extreme case where $k_{\mathrm{nr}}(\mathrm{TW})=0$, then the maximum value that $k_{r}\left(0^{+}\right)$ may assume from Eq. (7) is $1.0(2) \times 10^{8} \mathrm{~s}^{-1}$ and therefore, $k_{\mathrm{nr}}\left(0^{+}\right)=7.2(2) \times 10^{8} \mathrm{~s}^{-1}$. In the other unrealistic limits where $k_{r}\left(0^{+}\right)=0 \mathrm{~s}^{-1}, k_{\mathrm{nr}}\left(0^{+}\right)=8.23(1) \times 10^{8} \mathrm{~s}^{-1}$. The values for the $0^{-}$state follow immediately from similar expressions for $k_{F}\left(0^{-}\right) / k_{F}\left(0^{+}\right)$and are also listed. Clearly, from Table IX, conservative estimates for $k_{\mathrm{nr}}\left(0^{+}\right)$and $k_{\mathrm{nr}}\left(0^{-}\right)$are 7.7(5) $\times 10^{8}$ and $8.5(5) \times 10^{8} \mathrm{~s}^{-1}$, respectively, with a further restriction that $k_{\mathrm{nr}}\left(0^{+}\right) / k_{\mathrm{nr}}\left(0^{-}\right)=1.10(1)$. (Here, the ranges are specified as an error since values approaching the limits become increasingly unlikely.) Finally, we note that the radiative rate of $0.5 \times 10^{8} \mathrm{~s}^{-1}$ found for tropolone in cyclohexane is near the center of this range. ${ }^{44,46}$ The excited-state properties that impact the nonradiative decay behavior of tropolone will be addressed in a forthcoming article on tropolone- $\mathrm{H}_{2} \mathrm{O}$. 


\section{SUMMARY AND CONCLUSIONS}

High-resolution spectroscopic studies of jet-cooled tropolone and its isotopomers have permitted a detailed examination of the proton transfer dynamics in the zero-point levels of the ground and first electronically excited singlet states. In $S_{0}$, rovibrational spectra have been obtained for three symmetric and four asymmetric forms of tropolone. In $S_{1}$, the rotationally resolved spectra of the $0^{+}$and $0^{-}$states have been obtained.

In the ground electronic state, a linear inversion model predicts inversion barriers for the three symmetric isotopomers ${ }^{12} \mathrm{C}$, OD, and ${ }^{13} \mathrm{C}_{5}$, ranging between $1883 \mathrm{~cm}^{-1}$ for the parent isotopomer to $2288 \mathrm{~cm}^{-1}$ for tropolone-OD. Since the tunneling dynamics in tropolone involves the cooperative motion of all atoms, the isotope dependence of the barrier height, like the observed vibrational-mode-specific tunneling splittings in the $S_{0}$ and $S_{1}$ states, suggests the need to treat the tautomerization coordinate as a multidimensional surface that includes other vibrational degrees of freedom. ${ }^{11,12}$

Such mode-specific effects are also manifested in the observed changes in the coupling matrix elements and splittings of the asymmetric ${ }^{13} \mathrm{C}$ and ${ }^{18} \mathrm{O}$ forms, illustrating the extreme sensitivity of the dynamics to even the zero-point energies. The kinetic isotope effect impacts the zero-point energies of all vibrational modes to varying degrees depending on extent of atom participation in the 39 vibrational degrees of freedom. For ${ }^{13} \mathrm{C}_{4(6)}$ and ${ }^{13} \mathrm{C}_{3(7)}$, the magnitude of the change predicted from a simple two-state model amounts to $\approx 20 \%$ and $\approx 50 \%$ of the observed splitting. The tunneling dynamics of the ${ }^{12} \mathrm{C}_{2(1)}$ and ${ }^{18} \mathrm{O}$ isotopomers undergo drastic changes, however, where the tunneling process is essentially "turned off" as revealed by order-of-magnitude or more reductions in the off-diagonal matrix elements. From comparisons of the substituted atom positions with theoretical predictions, the isomeric forms favored in two lowest tunneling states of ${ }^{12} \mathrm{C}_{2(1)}$ are reversed relative to the other asymmetric forms. The calculated harmonic ZPE differences, while somewhat overestimated, confirm these results.

From rotationally resolved studies in $S_{1}$, the lifetimelimited linewidths have been used to place restrictive ranges on the radiative and nonradiative decay rates of tropolone when combined with previous quantum yield data on tropolone and tropolone- $\mathrm{H}_{2} \mathrm{O}{ }^{45}$ The rotational parameters, smooth line shapes, decay rates, and ratio determined for the two inversion levels reveal intrinsic properties needed to arrive at a better understanding of the $S_{1}$ photophysics of tropolone and its complexes.

\section{ACKNOWLEDGMENTS}

One of the authors (T.A.B.) is with the W. R. Wiley Environmental Molecular Sciences Laboratory, a national scientific user facility sponsored by the Department of Energy's Office of Biological and Environmental Research located at the Pacific Northwest National Laboratory. The Pacific Northwest National Laboratory is operated for the United States Department of Energy by Battelle under contract No. DE-AC05-76RLO 1830. The authors would like to extend special thanks to R. L. Redington for his helpful dis- cussions. One of the authors (J.C.K.) wishes to acknowledge the National Research Council for the postdoctoral fellowship support.

${ }^{1}$ M. A. Rios and J. Rodriguez, Can. J. Chem. 69, 201 (1991).

${ }^{2}$ K. Tanaka, H. Honjo, T. Tanaka, H. Kohguchi, Y. Ohshima, and Y. Endo, J. Chem. Phys. 110, 1969 (1999).

${ }^{3}$ R. K. Frost, F. C. Hagemeister, C. A. Arrington, D. Schleppenbach, T. S. Zwier, and K. D. Jordan, J. Chem. Phys. 105, 2605 (1996).

${ }^{4}$ R. L. Redington, T. E. Redington, and J. M. Montgomery, J. Chem. Phys. 113, 2304 (2000).

${ }^{5}$ R. L. Redington and R. L. Sams, J. Phys. Chem. A 106, 7494 (2002).

${ }^{6}$ R. L. Redington and T. E. Redington, J. Mol. Spectrosc. 78, 229 (1979). ${ }^{7}$ R. L. Redington, T. E. Redington, T. A. Blake, R. L. Sams, and T. J. Johnson, J. Chem. Phys. 122, 224311 (2005).

${ }^{8}$ M. V. Vener, S. Scheiner, and N. D. Sokolov, J. Chem. Phys. 101, 9755 (1994).

${ }^{9}$ J. J. Nash, T. S. Zwier, and K. D. Jordan, J. Chem. Phys. 102, 5260 (1995).

${ }^{10}$ S. Takada and H. Nakamura, J. Chem. Phys. 102, 3977 (1995); Z. Smedarchina, W. Siebrand, and M. Z. Sgierski, ibid. 104, 1203 (1996).

${ }^{11}$ K. Giese and O. Kuhn, J. Chem. Phys. 123, 054315 (2005).

${ }^{12}$ R. L. Redington, J. Chem. Phys. 113, 2319 (2000).

${ }^{13}$ A. C. P. Alves and J. M. Hollas, Mol. Phys. 23, 927 (1972).

${ }_{15}^{14}$ A. C. P. Alves and J. M. Hollas, Mol. Phys. 25, 1305 (1973).

${ }^{15}$ A. E. Bracamonte and P. H. Vaccaro, J. Chem. Phys. 120, 4638, (2004).

${ }^{16}$ R. L. Redington and C. W. Bock, J. Chem. Phys. 95, 10284 (1991).

${ }^{17}$ R. L. Redington, Y. Q. Chen, G. J. Scherer, and R. W. Field, J. Chem. Phys. 88, 627 (1988); R. L. Redington, T. E. Redington, M. A. Hunter, and R. W. Field, ibid. 92, 6456 (1990).

${ }^{18}$ H. Sekiya, Y. Nagasgima, and Y. Nishimura, Chem. Phys. Lett. 160, 581 (1989).

${ }^{19}$ H. Sekiya, Y. Nagasgima, and Y. Nishimura, J. Chem. Phys. 92, 5761 (1990).

${ }^{20}$ K. Nishi, H. Sekiya, H. Kawakami, A. Mori, and Y. Nishimura, J. Chem. Phys. 109, 1589 (1998).

${ }^{21}$ K. Nishi, H. Sekiya, H. Kawakami, A. Mori, and Y. Nishimura, J. Chem. Phys. 111, 3961 (1999).

${ }^{22}$ H. Sekiya, T. Tsuji, S. Ito, A. Mori, H. Takeshita, and Y. Nishimura, J. Chem. Phys. 101, 3464 (1994).

${ }^{23}$ T. Tsuji, H. Sekiya, Y. Nishimura, R. Mori, A. Mori, and H. Takeshita, J. Chem. Phys. 97, 6032 (1992).

${ }^{24}$ R. D. Suenram, J. U. Grabow, A. Zuban, and I. Leonov, Rev. Sci. Instrum. 70, 2127 (1999); A. R. Hight Walker, W. Chen, S. E. Novick, B. D. Bean, and M. D. Marshall, J. Chem. Phys. 102, 7298 (1995).

${ }^{25}$ D. F. Plusquellic, S. R. Davis, and F. Jahanmir, J. Chem. Phys. 115, 225 (2001).

${ }^{26}$ D. F. Plusquellic, R. J. Lavrich, T. Petralli-Mallow, S. R. Davis, T. M. Korter, and R. D. Suenram, Chem. Phys. 283, 355 (2002).

${ }^{27}$ H. M. Pickett, J. Chem. Phys. 56, 1715 (1972)

${ }^{28}$ H. M. Pickett, R. L. Poynter, E. A. Cohen, M. L. Delitsky, J. C. Pearson, and H. S. P. Muller, J. Quant. Spectrosc. Radiat. Transf. 60, 883 (1998).

${ }^{29}$ B. N. Taylor and C. E. Kuyatt, NIST Tech. Note 1297, 20402 (1994).

${ }^{30}$ J. T. Hougen and J. K. G. Watson, Can. J. Phys. 43, 298 (1965); A. Held, B. B. Champagne, and D. W. Pratt, J. Chem. Phys. 95, 8732 (1991).

${ }^{31}$ D. F. Plusquellic and D. W. Pratt, J. Chem. Phys. 97, 8970 (1992).

${ }^{32}$ W. A. Majewski, J. F. Pfanstiel, D. F. Plusquellic, and D. W. Pratt, in Laser Techniques in Chemistry, edited by A. B. Myers and T. R. Rizzo (Wiley, New York, 1995), Vol. XXIII; D. F. Plusquellic, R. D. Suenram, B. Maté, J. O. Jensen, and A. C. Samuels, J. Chem. Phys. 115, 3057 (2001).

${ }^{33}$ J. A. Hageman, R. Wehrens, R. de Gelder, W. L. Meerts, and L. M. C. Buydens, J. Chem. Phys. 113, 7955 (2000).

${ }^{34}$ W. H. Press, S. A. Teukolsky, W. T. Vetterling, and B. P. Flannery, Numerical Recipies in C: The Art of Scientific Computing (Cambridge University Press, Cambridge, England, 2001).

${ }^{35}$ Y. R. Wu and D. H. Levy, J. Chem. Phys. 91, 5278 (1989); G. Berden, W. L. Meerts, and E. Jalviste, ibid. 103, 9596 (1995).

${ }^{36}$ S. L. Baughcum, R. W. Duerst, W. F. Rowe, Z. Smith, and E. B. Wilson, J. Am. Chem. Soc. 103, 6296 (1981).

${ }^{37}$ S. L. Baughcum, Z. Smith, E. B. Wilson, and R. W. Duerst, J. Am. Chem. Soc. 106, 2260 (1984); P. Turner, S. L. Baughcum, S. L. Coy and Z. Smith, ibid. 106, 2265 (1984). 
${ }^{38}$ J. Kraitchman, Am. J. Phys. 21, 17 (1953).

${ }^{39}$ C. C. Costain, Trans. Am. Crystallogr. Assoc. 2, 157 (1966).

${ }^{40}$ E. B. Wilson, Jr., J. C. Decius, and P. C. Cross, Molecular Vibrations: The Theory of Infrared and Raman Vibrational Spectra (McGraw-Hill, New York, 1955).

${ }^{41}$ J. W. Ochterski, Vibrational Analysis in Gaussian, Technical Support Information, www.gaussian.com, 29 October 1999.

${ }^{42}$ H. F. Hameka and J. R. de la Vega, J. Am. Chem. Soc. 106, 7703 (1984).

${ }^{43}$ M. J. Frisch, G. W. Trucks, H. B. Schlegel, et al., Gaussian 03, Revision
B.04, Gaussian, Inc., Pittsburgh, PA, 2003.

${ }^{44}$ V. J. MacKenzie, H. K. Sinha, S. C. Wallace, and R. P. Steer, Chem. Phys. Lett. 305, 1 (1999).

${ }^{45}$ F. C. Hagemeister, C. A. Arrington, B. J. Giles, B. Quimpo, L. Zhang, and T. S. Zwier, in Cavity-Ringdown Spectroscopy: An UltratraceAbsorption Measurement Technique, ACS Symposium Series No. 720, edited by K. W. Busch and M. A. Busch (American Chemical Society, Washington, DC, 1999), Chap. 14, p. 203.

${ }^{46}$ V. J. MacKenzie, Ph.D. thesis, University of Saskatchewan, 1999. 